\title{
Ventajas del uso de sistemas de trazabilidad electrónica en procesos de manufactura
}

\author{
Jaime A. León-Duarte, Blanca M. De La Re-Iñiguez y Luis F. Romero-Dessens \\ Universidad de Sonora, Departamento de Ingeniería Industrial, Blvd. Luis Encinas J, 83000, Hermosillo, Sonora. México. \\ (correo-e: jaime.leon@unison.mx; blanca_melissadlr@hotmail.com; luisfelipe.romero@unison.mx)
}

Recibido Jul. 23, 2019; Aceptado Sep. 23, 2019; Versión final Oct. 10, 2019, Publicado Feb. 2020

\begin{abstract}
Resumen
El objetivo del estudio es desarrollar una metodología de mejora para un proceso de producción de arneses automotrices mediante la implementación de un sistema de monitoreo electrónico por código de barras, que permita obtener la trazabilidad de los productos procesados e identificar el origen, o causa raíz de los problemas de calidad. La metodología consta de un diagnóstico previo del área, el desarrollo y pruebas del programa, la implementación del sistema dentro del área, así como la capacitación del personal involucrado. Se encontraron los principales defectos del área de inyección de espuma, y con ayuda del sistema de trazabilidad se logró identificar las causas raíz de los mismos, por lo que se tomaron acciones específicas. Después de tres meses de implementación se redujo en $15 \%$ el porcentaje del principal defecto encontrado.
\end{abstract}

Palabras clave: sistemas de trazabilidad; trazabilidad electrónica; código de barras; RFID

\section{Advantages of the use of electronic traceability systems in manufacturing processes}

\begin{abstract}
The objective of this study is to develop a methodology for improving an automotive harness manufacturing process through the implementation of an electronic bar code monitoring system that allows obtaining the traceability of the processed products and identifying the origin of the quality problems. The methodology consists of an initial diagnosis of the area, the development and testing of the program, and the implementation of the system within the area, as well as the training of the personnel involved. The main defects of the area of foam injection were found, and with the help of the traceability system it was possible to identify the root causes of them, for which specific actions were taken. After three months of implementation the percentage was reduced by $15 \%$ of the main defect found.
\end{abstract}

Keywords: traceability systems; electronic traceability; barcode; RFID 


\section{INTRODUCCIÓN}

Las tecnologías de información y comunicación (TIC) crecen rápidamente, lo que brinda a distintas empresas oportunidades para integrarlas a su cadena de suministro (Musa y Dabo, 2016). Las TIC se constituyen principalmente por inversiones en equipos de cómputo, software y tecnología de comunicación. Existen múltiples estudios que demuestran como las TIC aumentan el desempeño y/o la productividad de las empresas de distintos sectores, como el estudio llevado a cabo en una institución educativa por Alcibar et al. (2018), o el estudio de Arévalo-Arecillas et al. (2018) en las empresas de servicios, y aunque estas investigaciones abarquen sectores distintos, es la industria de servicios la beneficiaria por la adopción de estas tecnologías (Alderete y Gutiérrez, 2012), reflejándose positivamente en su desempeño e innovación.

Las TIC son instrumentos estratégicos debido a su capacidad para modificar la estructura y los modelos de negocio de las empresas (Piñeiro et al., 2016), permitiendo a distintas organizaciones la posibilidad de mejorar su competitividad, así como la habilidad de incrementar su capacidad de respuesta mediante la adaptación de sus estrategias de operaciones, métodos y tecnologías a datos de negocio prácticamente en tiempo real (Musa y Dabo, 2016). Por lo anterior, actualmente es muy difícil, visualizar una organización exitosa que no se apoye en la tecnología para la administración de sus procesos (Saavedra y Tapia, 2013). La presente investigación muestra la aplicación del código de barras, como un sistema de trazabilidad, en un estudio de tipo cuantitativo explicativo, realizado en un proceso de recubrimiento de arneses por inyección de espuma de una empresa manufacturera, localizada en el noroeste de México, proveedora del sector automotriz y con clientes alrededor del mundo, principalmente fabricantes de maquinaria pesada.

Con el uso de las distintas TIC se busca contribuir en el fujo de información a través de la cadena de suministro, de una forma ágil y eficiente. Para conseguir este propósito, se emplean los sistemas de identificación de productos y captura de datos, como lo son las tecnologías de código de barras y radiofrecuencia. Es de gran importancia para las organizaciones identificar sus productos a lo largo la cadena de suministro debido a la cantidad de transacciones que se realizan diariamente, situación que genera el manejo de mucha información para la planeación y control de las operaciones de los involucrados en la cadena de suministro, como proveedores, productores, transportistas, distribuidores y clientes. Por lo tanto, se ofrece la trazabilidad de los productos como un valor agregado y como medio para satisfacer distintas normas y requerimientos legales (Correa et al., 2009).

La trazabilidad se considera una herramienta para la identificación y registro de información que facilita la mejora de procesos de control, sobre todo para aquellas empresas que buscan resultados confiables con errores mínimos dentro de sus procesos productivos. De esta manera, la incorporación de un sistema de trazabilidad en una organización se traduce en una mayor eficiencia en los procesos, menores costos por fallas o defectos y un mejor servicio al cliente, entre otros (Sosa, 2017). Hockenberger (2014), define trazabilidad como la habilidad para dar seguimiento y localización a productos, lotes o componentes a lo largo de la cadena de suministro, en los distintos procesos de una empresa, desde su primera etapa como materia prima (inclusive desde su origen con el proveedor), hasta que el producto terminado llega al consumidor final.

La Organización Internacional para la Estandarización (ISO), define la trazabilidad como "la capacidad para seguir el histórico, la aplicación o la localización de un objeto; al considerar un producto o un servicio, la trazabilidad puede estar relacionada con: el origen de los materiales y las partes; el histórico del proceso; y la distribución y localización del producto o servicio después de la entrega" (ISO 9000:2015). Los sistemas de seguimiento de productos existen desde antes de que se conociera el término de trazabilidad, tal es el caso de la industria ganadera, en donde la "marca de fuego" era el sistema que permitía conocer a quien pertenecía el ganado (Dassatti, 2015). Posteriormente se introdujeron las tecnologías que conocemos en la actualidad, como la RFID (tecnología por radiofrecuencia) y el código de barras; este último tuvo su primera aparición en el campo de ventas en junio de 1974, siendo un paquete de goma de mascar el primer escaneado para su venta (Hayat, 2012).

Dependiendo del sector en donde se apliquen los sistemas de trazabilidad, estos proveen a la empresa de distintas ventajas, como el incremento de la eficiencia operacional, debido a que el registro de información es más ágil y eficaz, así como el procesamiento de datos, esto ayuda a seguir el producto a su paso por la cadena de suministro de forma más eficiente y permite responder rápidamente ante cualquier anomalía que se pueda presentar, lo que ayuda reducir el tiempo de trabajo del personal de la organización ya que permite identificar problemas de forma específica, sin la necesidad de consultar con todo el personal involucrado en el proceso bajo estudio (Rădulescu y Popescu, 2014). Las empresas buscan constantemente mejorar la calidad en sus productos y en sus procesos, por lo que implementan distintas herramientas para encontrar las causas raíz de los problemas como el diagrama de Pareto o el diagrama de Ishikawa (Luca et al., 2017). Las tecnologías de trazabilidad permiten identificar en que parte del proceso ocurre un defecto, y con el apoyo de la información que estos sistemas brindan al usuario es posible identificar causas raíz de una forma más rápida. 


\section{TECNOLOGÍAS DE RADIOFRECUENCIA}

Uno de los sistemas de trazabilidad más utilizados en la industria actual, es la tecnología por radiofrecuencia o RFID (por sus siglas en inglés), el cual es un sistema de identificación y adquisición de información, que permite recolectar y transferir datos de producción y negocios mediante ondas de radiofrecuencia (Correa et al., 2010). Un sistema RFID se compone de una etiqueta electrónica que cumple la función de transmisor y de receptor de señal, ambos apoyados en un sistema decodificador (middleware); los datos de identificación son almacenados en la etiqueta y son transferidos al receptor de señal mediante ondas de radiofrecuencia, esto con la finalidad de que el sistema procese los datos recibidos para la identificación y monitoreo del producto a través de la cadena de suministro, o dentro de un proceso de manufactura (Musa y Dabo, 2016; Hozak y Collier, 2008).

El uso de la tecnología RFID permite a las distintas empresas identificar, clasificar y administrar el flujo de materiales e información de manera automática e inalámbrica a través de la cadena de suministro, sin necesidad de intervención humana lo que previene múltiples errores (Chen et al., 2013; Wang et al., 2010). Esta tecnología ofrece al usuario la trazabilidad en tiempo real, no sólo de sus productos, sino también de otros objetos, camiones, e inclusive del personal de la organización, entre muchos otros elementos que pueden ser identificados y seguidos de forma automática (Xu et al., 2013). Tajima (2007) agrupa los beneficios que los sistemas RFID como se muestra en la figura 1, divididos según el rol que se juega en la cadena de suministro, ya sea como proveedor, distribuidor o minorista, así como los beneficios que se pueden encontrar en cualquiera punto de la cadena de suministro.

\footnotetext{
Beneficios a través de la cadena de suministro

(1) Reducción de la merma

(2) Reducción del manejo de materiales

(3) Incremento de la precisión de los datos

(4) Gestión más rápida de excepciones

(5) Mejora el intercambio de información
}

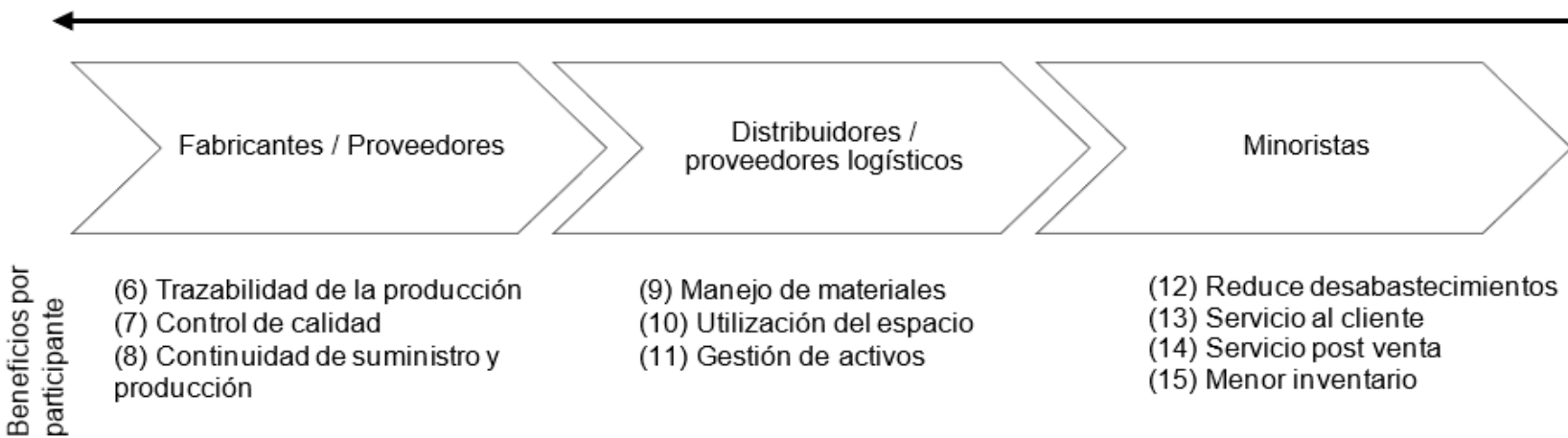

Fig. 1: Beneficios del RFID a través de la cadena de suministro (Traducida de Tajima 2007)

Chen et al. (2007) sintetiza los componentes que conforman un sistema RFID como se muestra en la figura 2 , el cual consta de una etiqueta RFID adherida al objeto que se dará seguimiento, un lector RFID, un sistema decodificador de información (middleware), un servidor con la información electrónica del código del producto, y los distintos sistemas de información de la empresa.

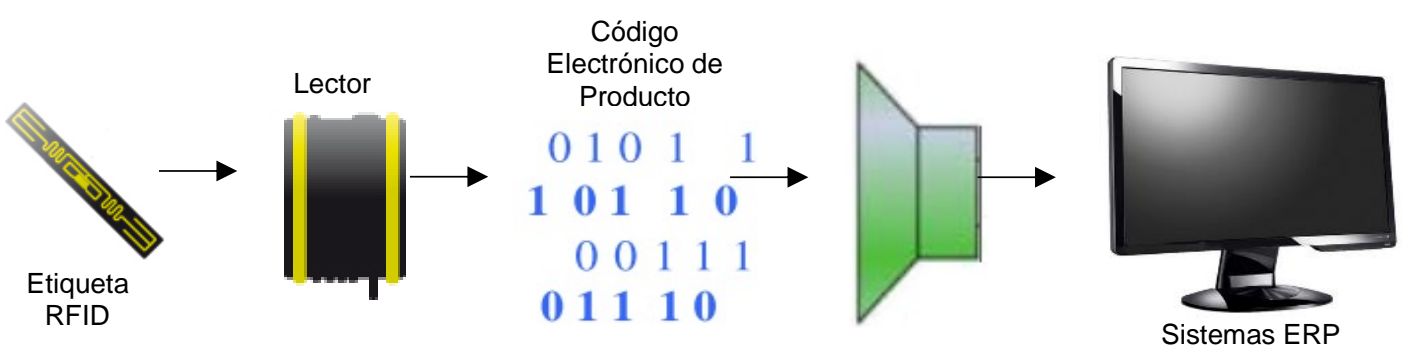

Fig. 2: Componentes de un sistema RFID (Adaptada de Chen et al. 2007) 


\section{Código de barras}

Uno de los sistemas de trazabilidad más utilizados es el código de barras, utilizado en cualquier tipo de sociedad, industria o mercado (Rădulescu y Popescu, 2014), esto gracias a su rapidez para escanear productos en comparación con otros sistemas, además de la gran velocidad de captura de información y su creciente popularidad en distintos sectores (Wasule y Metkar, 2017). Un sistema por código de barras le permite al usuario identificar productos, de manera automática e inequívoca; se utiliza para identificar números de productos, números seriales y números de lote. Este sistema juega un rol de gran importancia ya que permite a las compañías rastrear automáticamente sus productos a través de la cadena de suministro (Correa et al., 2010).

Barry (2011) señala como beneficios de los sistemas de código de barras: 1) captura de datos más rápida y precisa, reduciendo papeleo, errores y costos; 2) información actualizada; 3) medición de la productividad; 4) reducción en el tiempo de entrenamiento; 5) mejora la toma de decisiones, entre otros. Sin embargo, Hayat (2012) resume sus beneficios en tres principales categorías: 1) reducir los errores al ingresar datos. Una persona cometerá un error cada 300 veces que teclee información, y los códigos de barra, se estiman un error cada millón de capturas; 2) reducir el tiempo de inspección. Es más rápido encontrar un componente específico identificado con código de barras, en vez de buscarlo visualmente, ya que tardará más tiempo y es posible confundir el componente; 3 ) incrementar la trazabilidad. Incluye la información del operador, máquina, número de lote, vendedor, entre otros datos. Los componentes que integran un sistema de código de barras pueden variar según el campo donde se esté aplicando, Li et al. (2003) señalan los componentes básicos de un sistema, los cuales consisten en un escáner, etiquetas para el producto con el código de barras, una computadora y un software para el procesamiento de datos, como se muestra en la figura 3.

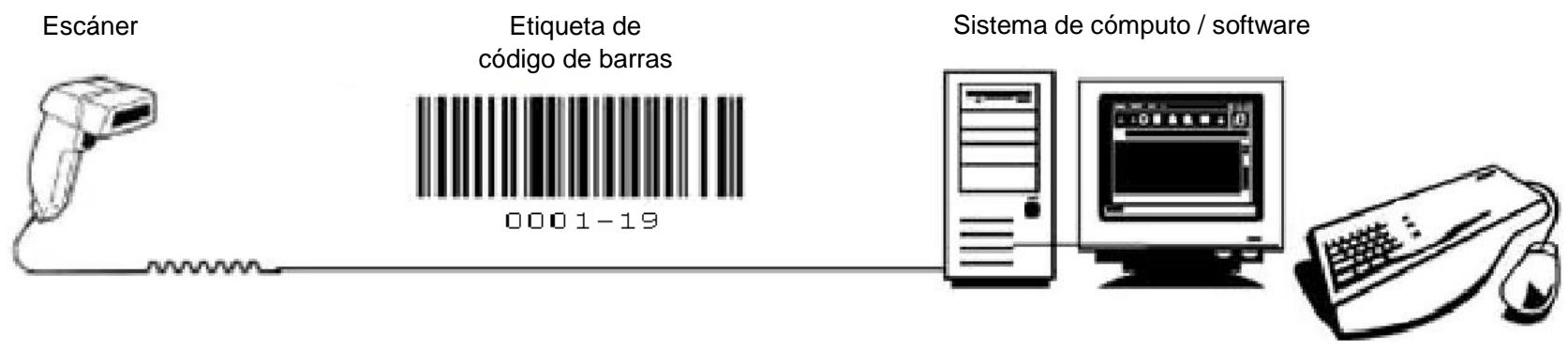

Fig. 3: Componentes de un sistema de código de barras (adaptada de Li et al, 2003).

Las dos tecnologías descritas, código de barras y RFID, cumplen con el mismo propósito de brindar trazabilidad a un producto o proceso sin embargo la elección de una tecnología o la otra, depende de las necesidades de la empresa, Li (2012) y Hozak y Collier (2008) realizan una comparación entre ambas tecnologías, la cual se sintetiza en la tabla 1.

Tabla 1: Comparación de las tecnologías de Código de Barras y RFID. (Adaptada de Li, 2012; Hozak y Collier, 2008)

\begin{tabular}{|c|c|c|}
\hline Atributo & Código de Barras & RFID \\
\hline Tecnología & Tecnología de imagen óptica & Inalámbrica, de radiofrecuencia \\
\hline Capacidad de datos & $\begin{array}{l}\text { Hasta } 24 \text { caracteres en códigos lineales y } \\
\text { hasta } 2000 \text { para bidimensionales }\end{array}$ & Miles de caracteres \\
\hline $\begin{array}{l}\text { Requerimientos de } \\
\text { lectura }\end{array}$ & Se requiere una línea de visión & $\begin{array}{l}\text { Requiere estar dentro del rango de } \\
\text { detección }\end{array}$ \\
\hline Durabilidad & $\begin{array}{l}\text { Sujeta a daños, remoción y uso; no se } \\
\text { puede leer si está sucia }\end{array}$ & $\begin{array}{l}\text { Alta, puede estar sujeta a impacto } \\
\text { ambiental. }\end{array}$ \\
\hline Seguridad & $\begin{array}{l}\text { Puede ser fácilmente reproducida la } \\
\text { información }\end{array}$ & $\begin{array}{l}\text { La información puede ser encriptada y } \\
\text { borrada }\end{array}$ \\
\hline Tasa de lectura & Lenta, una a la vez & Se leen varias etiquetas simultáneamente \\
\hline Costos & $\begin{array}{l}\text { La etiqueta cuesta menos de un centavo de } \\
\text { dólar, y muchas compañías ya cuentan con } \\
\text { la infraestructura necesaria. }\end{array}$ & $\begin{array}{l}\text { Puede costar entre } 10 \text { centavos y } 50 \\
\text { dólares cada una dependiendo de su } \\
\text { capacidad. }\end{array}$ \\
\hline
\end{tabular}




\section{Uso de la tecnología de trazabilidad}

Los sistemas de trazabilidad pueden ser utilizados en una amplia gama de sectores, como comúnmente se usa para la identificación de productos, hasta el seguimiento, de camiones, personas, e inclusive para rastrear boletos de algún evento deportivo, musical, cultural o social. La industria alimenticia requiere especial cautela y precisión, debido a que una enfermedad derivada de un mal cuidado de los alimentos puede terminar en una demanda y clausura de la empresa en cuestión. Continuamente se aprueban nuevas leyes que protegen al consumidor ya que obligan a las compañías productoras de alimentos que sus productos y procesos tengan los más altos estándares de calidad, y un sistema de trazabilidad apoyo en gran medida esta causa. Un de lo anterior, es el caso que presenta Rickard (2011), en donde se concreta una ley en los Estados Unidos que obliga a los productos de alimentos a incluir un sistema de trazabilidad en sus procesos como protección al consumidor.

Cuando se habla de una industria en la que está en riesgo la salud, o incluso la vida del consumidor, como es el caso de la industria farmacéutica, donde deberá incluirse un sistema de trazabilidad que permita detectar oportunamente cualquier anomalía. Hockenberger (2014) realiza un análisis de la importancia de un sistema de trazabilidad en la cadena de suministro de un medicamento, desde su lote de producción, hasta el punto de venta. Entre los beneficios que menciona este autor resaltan los siguientes: 1) cumplir con los requerimientos legales, 2) presenta una ventaja competitiva, 3) ayuda en el manejo de residuos, 4) protege a la marca de daños al poder probar la procedencia de sus productos y, 4) facilita en caso de que sea necesario pedir un lote de regreso por alguna anomalía.

De forma similar a la industria farmacéutica, cuando se trabaja en el sector hospitalario un error puede causar estragos en la vida de una persona, por lo que los sistemas de trazabilidad pueden ser encontrados en distintos sectores o procesos dentro de un hospital. Wang et al. (2016) implementan en un hospital un sistema de código de barras para apoyar con la medicación de los pacientes, con la finalidad de aumentar la seguridad de los pacientes y de reducir los errores del personal, esto debido a que son muchos paciente que cada doctor y enfermero atiende diariamente, lo que puede provocar confusión de las instrucciones para cada uno de ellos. Después de la implementación del sistema se logró agilizar el proceso de medicación.

En la industria manufacturera es importante conservar un alto nivel de calidad si se pretende alcanzar un alto grado de competitividad con las otras empresas. Día con día se busca agilizar procesos, eliminar errores, reducir defectos, y producir con la mejor calidad posible, por lo que para cumplir con este objetivo es necesario contar con un sistema de trazabilidad que apoye el seguimiento del producto a través de la cadena de suministro. En una empresa manufacturera de persianas, Ramírez-Hernández y Cuamea-Cruz (2014) muestran la simulación para la implementación de un sistema RFID, desde el almacén de materia prima y a través de cada uno de los procesos de transformación del producto, considerando defectos y re-trabajos, esto con la finalidad de brindar a la compañía una transparencia total en cuanto al paso de cada producto dentro de la empresa.

Un ejemplo sencillo para la aplicación de un sistema de código de barras en el sector educativo es el que proponen Rădulescu y Popescu (2014) para la biblioteca de la Universidad de Petróleo y Gas de Ploiești, en Rumania, con la finalidad de ayudar a los estudiantes a agilizar el proceso de solicitud de un préstamo de libro, y a los encargados de la biblioteca a minimizar sus esfuerzos reduciendo su trabajo. El sistema contiene información de los libros, su localización, los libros prestados y en existencia, las multas por entregas tardías por alumno, así como la información general de cada estudiante. Este sistema favorece la colaboración entre biblioteca y alumno, reduce los tiempos y mano de obra necesarios para su operación, como en el caso de los inventarios, en donde antes se requería de 25 personas trabajando por una semana, pero con la implementación del sistema de códigos de barras, sólo son necesarias 4 personas trabajando entre 4 y 5 horas.

Para que cualquier empresa funcione de una forma eficiente, es necesario tener un buen sistema de gestión de almacenes. El apoyo de un sistema de trazabilidad resulta fundamental, pero es necesario aplicarlo de la forma correcta. En el estudio realizado por Fachri et al. (2018) se realiza una evaluación para la implementación de un sistema RFID como parte del sistema de gestión de almacenes de una empresa en Indonesia. Se concluye con una notable mejora del proceso de selección de materia prima dentro del almacén, así como de facilitar el trabajo realizado diariamente por el personal, además de incrementar la productividad del almacén y modernizar el proceso de trabajo.

\section{CASO DE APLICACION}

En una industria manufacturera de arneses localizada al Noroeste de México, se producen piezas para clientes alrededor del mundo, que incluyen motocicletas, automóviles, camiones y maquinaria pesada. Del 
total de la producción de la empresa, cerca de la tercera parte de los arneses debe llevar un recubrimiento especial de espuma debido a las funciones que debe cumplir dentro del vehículo, la cual funciona como un aislamiento térmico. El proceso de inyección de espuma consiste en: 1) preparar un molde rociándolo con cera para que la pieza salga con facilidad al terminar el ciclo; 2) colocar la pieza en el molde e inyectar la mezcla; 3) esperar a que la mezcla se solidifique; 4) remover la pieza y retirar excesos de espuma.

Dentro del área de inyección de espuma, según el reporte diario de material defectuoso, se ha gastado cerca del $80 \%$ del presupuesto para defectos diariamente, y se proyecta que se rebase en los próximos meses. Los problemas del área van desde la subutilización de la mano de obra y maquinaria, faltar de capacidad para procesar piezas, y falta de organización. Actualmente existe control de las piezas procesadas, la cantidad de defectos, y la eficiencia del proceso. Sin embargo, gran cantidad de parámetros se desconocen puntualmente, y en ocasiones los datos que se obtienen no coinciden con la realidad del proceso, esto debido a que la captura de información es realizada por distintas personas, lo que facilita la posibilidad de cometer errores. Además, se desconoce cuándo y dónde ocurrió cada defecto, así como el responsable de este.

Con la finalidad de brindar una solución, se implementó, a inicios del 2019, un sistema de trazabilidad, con tecnología de código de barras. Se eligió esta opción sobre los sistemas RFID, principalmente por que la empresa ya cuenta con la infraestructura para esta tecnología debido a que actualmente utiliza estos códigos en otras áreas, sin embargo, en el proceso de inyección de espuma estos códigos no son utilizados. Por tal motivo la inversión se reduce considerablemente, ya que no fue necesaria la impresión de etiquetas, ni la adquisición de nuevas impresoras.

Con la implementación de este nuevo sistema de trazabilidad, se controló de manera más efectiva la producción del área en tiempo real, lo que permitió conocer los productos que entran y salen del área, la eficiencia por molde y los defectos por estación, entre otros beneficios. Con la información obtenida, los ingenieros responsables del área pueden tomar acciones específicas según operador, estación, molde o inclusive por la hora del día. En un mediano plazo, la implementación de este sistema se traducirá en una reducción del número de defectos y el consiguiente aumento de la eficiencia general del área. Para la implementación del sistema de código de barras se siguió una metodología de tres etapas, de diagnóstico, desarrollo de software e implementación, como se describen a continuación.

Etapa de diagnóstico: Se inició con un análisis del área y se llevó registro de los defectos durante 8 meses, se agruparon los datos y se creó una nueva clasificación de los mismos para ser identificados por códigos, los cuales fueron incluidos en el desarrollo del programa.

Etapa de desarrollo: Con la información obtenida se determinaron las variables a considerar para el desarrollo del programa, como la información de cada pieza, del operador, molde, estación, día y hora de producción. Se comenzó el desarrollo del sistema y se realizaron múltiples pruebas para comprobar su funcionalidad.

Etapa de implementación: Se instaló el equipo necesario y el programa dentro del área de inyección de espuma y se capacitó a todo el personal involucrado en el proceso, se les mostró como operar el programa para escanear una pieza, y se explicó cómo se generaban los reportes. Se procedió a utilizar el programa diariamente, escaneando cada uno de los productos procesados, los defectos quedaban registrados con la información del operador, la hora y la estación en la cual se trabajó.

\section{RESULTADOS}

Gracias a la implementación del sistema, se detectaron los principales defectos, identificando las causas de los mismos por lo que se tomaron acciones correctivas de forma inmediata. Se observó que uno de los principales defectos se debía a errores de los trabajadores, por lo que se procedió a impartir una nueva capacitación para el manejo de material y el proceso de inyección de espuma. Si un operador seguía ocasionando defectos por el mismo motivo, se procedió a tomar medidas disciplinarias. Además, en el proceso de selección de personal, los nuevos operadores pasaron por una capacitación más rigurosa y de un monitoreo constante durante las primeras semanas de operación.

De la misma forma, se encontró que otra de las principales causas del mismo defecto (y de otros en el área) era debido a una mala mezcla de material, por lo que se realizaron distintas pruebas con la calidad del material para reducir sus variaciones. El impacto de estas acciones no fue muy relevante debido a que la mezcla del material se ve afectada por múltiples factores, y algunos de ellos no pueden ser controlados con facilidad, como las condiciones climáticas y ambientales. Además, de los defectos encontrados en el área, se detectó que el $63 \%$ correspondía al segundo turno de producción (contra $37 \%$ del primer turno), principalmente por una supervisión deficiente y personal mal capacitado. Por tal motivo, se asignó a una persona encargada del monitoreo del segundo turno, además de brindar una capacitación general a los trabajadores del área. 
En un mes se presentó una mejoría en el área, desde la disciplina de los trabajadores, hasta la reducción en un $15 \%$ del principal defecto encontrado, según el reporte del sistema. Por lo tanto, se recomienda a la empresa continuar con el sistema, y buscar las causas raíz de sus defectos y tratarlos poco a poco para seguir mejorando. De los principales defectos que se encontraron, y sobre los que se tomaron acciones, se sintetiza en la tabla 2 la reducción de cada uno de ellos, después de 3 meses de implementar el sistema de código de barras dentro del área de inyección de espuma.

Tabla 2: Reducción de defectos principales

\begin{tabular}{|l|l|l|}
\hline Defecto & Situación anterior & Mejora \\
\hline Cobertura incompleta & $33 \%$ del total & Reducción del $15 \%$ \\
\hline Exceso de mezcla & $20 \%$ del total & Reducción del $7 \%$ \\
\hline Cable prensado & $17 \%$ del total & Reducción del $5 \%$ \\
\hline
\end{tabular}

\section{DISCUSION FINAL}

El desarrollo de un programa para trazabilidad dentro del área de inyección de espuma es comparable con la aplicación móvil implementada por Meza-Leal et al. (2017) para la trazabilidad interna en el proceso del café desde su producción hasta la cosecha. Ambas plataformas capturan la información relacionada a los procesos, con la finalidad de proporcionar mayor información al usuario y apoyar la toma de decisiones. Con la tecnología de código de barras que se implementó en el área, se logró el objetivo básico de los sistemas de trazabilidad, que es el dar seguimiento al producto a lo largo de la cadena de suministro, o en el caso particular del estudio, al interior de un proceso de inyección de espuma. Con el apoyo del sistema, el staff de ingeniería es capaz de tomar decisiones con base en información real, por lo que en un mediano plazo se observan mejoras en el área, como se describió anteriormente. La organización ya utilizaba un sistema de código de barras, sin embargo este no se aprovechaba dentro del proceso de inyección de espuma, pero gracias a la existencia de una plataforma similar y de la infraestructura necesaria, fue relativamente sencillo realizar la implementación. Dentro de la compañía, el área de inyección de espuma representaba el segundo lugar de las áreas que generaban mayores costos por defectos, situación que ha mejorado, por lo que, según el grado de implementación y seguimiento por parte de ingeniería, se estima una reducción mayor a futuro, con un proceso estable y adaptado al sistema de trazabilidad y monitoreo de calidad.

\section{CONCLUSIONES}

De acuerdo al trabajo presentado y a los resultados obtenidos, se pueden plantear las siguientes conclusiones principales: 1.- La implementación del sistema de trazabilidad por código de barras, contribuyó a la reducción de defectos presentados en el área, así como a la reducción de los costos de la no calidad. 2.- Al tomarse las acciones correctivas y luego de un mes de su implementación se encontraron reducciones del $15 \%, 7 \%$ y $5 \%$ respecto a la cobertura incompleta, exceso de mezcla y cable prensado respectivamente. 3.- Debido a que el estudio se desarrolló en la cuarta parte del área para evaluar el funcionamiento del sistema, se propone extender la implementación del sistema a toda el área de inyección de espuma para obtener un mayor impacto en lo referente a los costos por productos defectuosos

\section{REFERENCIAS}

Alcibar, M.F.; A. Monroy, y M. Jiménez, Impacto y Aprovechamiento de las Tecnologías de la Información y las Comunicaciones en la Educación Superior, DOI 10.4067/S0718-07642018000500101, Información Tecnológica, 9 (5), 101-110 (2018).

Alderete, M. y L. Gutiérrez, TIC y Productividad en las Industrias de Servicios en Colombia, ISSN 0120-2596, Lecturas de Economía, 77, 163-188 (2012).

Arévalo-Avecillas, D.; S. Nájera-Acuna, y E.A. Piñero, La Influencia de la Implementación de las Tecnologías de Información en la Productividad de Empresas de Servicios, DOI: 10.4067/S0718-07642018000600199, Información Tecnológica, 29 (6), 199-212 (2018).

Barry, C., How to: Leverage Barcode Technology, ISSN: 1554-8961, Multichannel Merchant, 7(3), 38-40 (2011).

Chen, J.C., y otros 5 autores, Warehouse Management with Lean and RFID Application: A Case Study, DOI 10.1007/s00170-013-5016-8, International Journal of Advanced Manufacturing Technology, 69, 531-542 (2013).

Chen, J.L., y otros 3 autores, Architecture Design and Performance Evaluation of RFID Object Tracking Systems, DOI:10.1016/j.comcom.2007.04.003, Computer Communications, 30(9), 2070-2086 (2007).

Correa, A.A., R.A. Gómez y J.A. Cano, Gestión de Almacenes y Tecnologías de la Información y Comunicación (TIC), ISSN 0123-5923, Estudios Gerenciales, 26(117), 145-171 (2010). 
Correa, A., C.E. Álvarez y R.A. Gómez, Sistemas de Identificación por Radiofrecuencia, Código de Barras y su Relación con la Gestión de la Cadena de Suministro, ISSN 0123-5923, Estudios Gerenciales, 26(116), 115-141 (2009).

Dassatti, G., Trazabilidad de la Carne, Bioseguridad y Seguridad Nacional. El sistema de cajas negras, ISSN: 1022-1301, Archivos Latinoamericanos de Producción Animal, 23(5), 23-29 (2015).

Fachri, S., R. Maulana y B. Rahmad, Qualitative Evaluation of RFID Implementation on Warehouse Management System, DOI 10.12928/TELKOMNIKA.v16i3.8400, TELKOMNIKA, 16(3), 1303-1309 (2018).

Hayat, K., Linking Barcode Technology To Quality Control, ISSN: 0360-9936, Quality, 51(7), 57-60 (2012).

Hockenberger, S., Track and trace: Business Benefits Beyond Compliance, DOI 10.1038/507008b, Supply \& Demand Chain Executive, 507(7490), 18-21 (2014).

Hozak, K. y D.A. Collier, RFID as an Enabler of Improved Manufacturing Performance, DOI 10.1111/j.15405915.2008.00214.x, Decision Sciences, 39(4), 859-882 (2008).

ISO 9000: 2015, Sistemas de Gestión de la Calidad, 24, Ginebra, Suiza, (2015).

Li, H., Z. Chen y C.T.C. Wong, Barcode Technology for an Incentive Reward Program to Reduce Construction Wastes, DOI: 10.1111/1467-8667.00320, Computer-Aided Civil and Infrastructure Engineering, 18, 313-324 (2003).

Li, Z., Characterization and Performance Analysis of UHF RFID Tag for Environment Sensing Applications. M.C. University of Waterloo (2012).

Luca, L. y M. Pasare y A. Stancioiu, Study to Determine a New Model of the Ishikawa Diagram for Quality Improvement, ISSN 1844 - 640X, Fiability \& Durability, 1, 249-254 (2017).

Meza-Leal, J., N. Farías-Mendoza y R. Chávez-Valdez, Trazabilidad de la Producción y Cosecha del Café Orgánico implementado con Tecnologías Móviles, ISSN: 2007-9753, Revista Internacional de Investigación e Innovación Tecnológica, 5 (27), 1 - 17 (2017).

Musa, A. y A.A.A. Dabo, A Review of RFID in Supply Chain Management: 2000-2015, DOI 10.1007/s40171-016-0136-2, Global Journal of Flexible Systems Management, 17(2), 189-228 (2016).

Piñeiro, C., P. de Llano y M. Rodríguez, Las TIC como Inductores de Competitividad y Facilitadores del Éxito Empresarial, ISSN: 2387-0184, International Journal of Information Systems and Software Engineering for Big Companies, 3(1), 8-26 (2016).

Rădulescu, G. y C. Popescu, About Barcode Technology Case study: Computerization of a Library, ISSN: 1224-8495, Petroleum - Gas University of Ploiesti Bulletin, 66(3), 7-14 (2014).

Ramírez-Hernández, R. y G. Cuamea-Cruz, Real-time Production Visibility Protocol using Radiofrequency Identification Technology at a Blinds Manufacturing Company, DOI: 10.30958/ajte.1-1-5, Athens Journal of Technology Engineering, 1(1), 63-72 (2014).

Rickard, D., Traceability Deep impact, ISSN: 0027-996X, National Provisioner, (July), 44-48 (2011).

Saavedra, M.L. y B. Tapia, El Uso de las Tecnologías de Información y Comunicación TIC en las Micro, Pequeñas y Medianas Empresas (MIPyME) Industriales Mexicanas, ISSN: 1690-7515, Revista Venezolana de Información, Tecnología y Conocimiento, (1), 85-104 (2013).

Sosa, C.I., Propuesta de un Sistema de Trazabilidad de Productos para la Cadena de Suministro Agroalimentaria. Univesidad Politécnica de Valencia (2017).

Tajima, M., Strategic Value of RFID in Supply Chain Management, DOI 10.1016/j.pursup.2007.11.001, Journal of Purchasing \& Supply Management, 13, 261-273 (2007).

Wang, B.N.T., P. Brummond y J.G. Stevenson, Comparison of Barcode Scanning by Pharmacy Technicians and Pharmacists' Visual Checks for Final Product Verification, DOI 10.2146/ajhp150135, American Journal of Health-System Pharmacy, 73(2), 69-75 (2016).

Wang, H., S. Chen e Y. Xie, An RFID-Based Digital Warehouse Management System in the Tobacco Industry: A Case Study, DOI: 10.1080/00207540903564918, International Journal of Production Research, 48(9), 2513-2548 (2010).

Wasule, S. y S. Metkar, Improvement in Two-dimensional Barcode. DOI 10.1007/s12046-017-0670-4, Sadhana, 42(7), 1025-1035 (2017).

Xu, Z., y otros 5 autores, Management Optimisation Based on Dynamic SKU for RFID-enabled Warehouse Management in the Steel Supply Chain. DOI 10.1080/00207543.2012.751513, International Journal of Production Research, 51(10), 2981-2996 (2013). 